\title{
Review: cannabinoids and codeine have similar effects on pain relief, but cannabinoids commonly cause psychotropic adverse effects
}

Campbell FA, Tramèr MR, Carroll D, et al. Are cannabinoids an effective and safe treatment option in the management of pain? A qualitative systematic review. BMJ 2001 Jul 7;323:13-6.

\section{QUESTION: What are the effectiveness and safety of cannabinoids for pain management?}

\section{Data sources}

Full publications of studies were identified by searching Medline (1966 to 1999), EMBASE/Excerpta Medica (1974 to 1999), the Oxford Pain Database (1950 to 1994), and the Cochrane Library (1999, Issue 3) with the terms marijuana, marihuana, mariuana, cannabis, cannabinoids, tetrahydrocannabinol (THC), delta-9-THC, nabilone, pain, analgesia, and random. Bibliographies of relevant studies were scanned.

\section{Study selection}

Studies were selected if they were randomised controlled trials (RCTs) that compared cannabis or cannabinoids with an analgesic agent or placebo for pain management. Studies of experimental pain were excluded.

\section{Data extraction}

Data were extracted on study quality, interventions, and outcomes.

\section{Main results}

20 RCTs were identified, 9 of which met the selection criteria (222 patients). The types of cannabinoids used were oral delta-9-THC 5 to $10 \mathrm{mg}$; an oral synthetic nitrogen analogue of THC $4 \mathrm{mg}$; oral benzopyranoperidine
2 to $4 \mathrm{mg}$; and intramuscular levonantradol 1.5 to $3 \mathrm{mg}$. No study assessed cannabis or other inhaled or smoked cannabinoids. The other active treatment drugs were oral codeine 50 to $120 \mathrm{mg}$ and oral secobarbital $50 \mathrm{mg}$. Studies varied in interventions and regimens, clinical settings, duration of follow up, and outcomes. 5 studies were on cancer pain; 2 were on chronic, non-malignant pain; and 2 were on postoperative pain. In 7 studies, follow up was 6 to 7 hours; in 1 study, follow up was 6 weeks; and in 1 study, follow up was 5 months. In patients with cancer pain, oral delta-9-THC and an oral synthetic nitrogen analog of THC were as effective as codeine, but oral benzopyranoperidine was less effective than codeine. In patients with postoperative pain, intramuscular levonantradol was more effective than placebo, and adverse effects with levonantradol were common but mild. In all studies, adverse effects (often psychotropic) were common.

\section{Conclusion}

Cannabinoids and codeine have similar effects on pain relief, but cannabinoids commonly cause psychotropic adverse effects.
Sources of funding: Swiss National Research Foundation and Royal College of Nursing Institute.

For correspondence: $M s$ F A Campbell, Pain Management Centre Undercroft, South Block, Queen's Medical Centre, Nottingham NG7 2UH, UK. Fax +441159709196.

\section{COMMENTARY}

Studies done in the previous few years have suggested that cannabinoids may be effective in certain populations for alleviating nausea and symptoms of anorexia, although their clinical indications have been unclear because of the high incidence of psychotropic side effects and the lack of sound trials comparing cannabinoids with standard treatment. ${ }^{2}$ Despite the lack of clear evidence on safety and efficacy, a survey done in 1994 showed that most physicians in the UK wished to have cannabinoids available by prescription. In this setting, Campbell et al and Tramèr et al completed their respective systematic reviews, which examined the efficacy of cannabinoids in the treatment of pain and chemotherapy-induced nausea and vomiting.

Cannabinoids in pain management provide analgesic effects similar to those of codeine. Cannabinoids commonly cause psychotropic side effects, but whether these effects are more common than with codeine is uncertain. At present, insufficient evidence exists to support the widespread use of cannabinoids over currently available analgesic treatments for pain management. However, the effects of inhaled cannabinoids were not assessed in this analysis.

The findings regarding chemotherapy-induced nausea and vomiting were somewhat more intriguing: cannabinoids were shown to be slightly superior to such conventional antiemetics as prochlorperazine or metoclopramide. Although psychotropic side effects remained common, some of these effects, including euphoria and sedation, may have been perceived as beneficial. Many patients reported having a preference for cannabinoids, but whether this preference was because of beneficial side effects was not evaluated.

What is to be gained from these 2 reviews? The review by Campbell $e t$ al assessing the efficacy of cannabinoids in the treatment of pain puts to rest the question of whether these oral or intramuscular agents are useful in that setting. Clearly, the review of published trials shows that unless more potent and less toxic cannabinoid derivatives are developed and studied, these agents are unlikely to have any important role as therapeutic options in pain management.

The results of the review by Tramèr et al assessing the efficacy of cannabinoids in the treatment of nausea and vomiting are less clear. One can certainly argue that any relevance of the "slightly superior" outcomes of treatment with cannabinoids over conventional antiemetics may be offset by the concerns about the psychotropic side effects that routinely accompany the use of these agents. However, as pointed out in Belknap's commentary ${ }^{4}$ on the Voth study, ${ }^{1}$ no clinical trials were reported comparing cannabinoids to 5-hydroxytryptamine-3 receptor antagonists, which have become the standard of care in the prevention of chemotherapy-induced nausea and vomiting. Until such trials are done, the use of cannabinoids for this indication remains uncertain.

continued on next page 


\section{Review: cannabinoids control chemotherapy-induced nausea and vomiting but increase the risk of side effects}

Tramèr MR, Carroll D, Campbell FA, et al. Cannabinoids for control of chemotherapy induced nausea and vomiting: quantitative systematic review. BMJ 2001 Jul 7;323:16-21.

\section{QUESTION: In patients with chemotherapy-induced sickness, what are the antiemetic efficacy and adverse effects of cannabinoids?}

\section{Data sources}

Full publications of studies were identified by searching Medline (from 1966), EMBASE/Excerpta Medica (from 1982), and the Cochrane Library (2000, Issue 3) with the terms cannabinoids, cannabis, nabilone, tetrahydrocannabinol (THC), THC, marihuana, marijuana, levonantradol, dronabinol, randomized, and human. Bibliographies of relevant studies were checked.

\section{Study selection}

Studies were selected if they were randomised controlled trials (RCTs) comparing the antiemetic efficacy of cannabis with any other antiemetic agent or placebo in patients having chemotherapy.

\section{Data extraction}

Data were extracted on study quality, patients, interventions and regimens, and outcomes.

\section{Main results}

30 RCTs (1366 patients) published between 1975 and 1997 met the selection criteria. The mean number of patients per study was 46 (range 8 to 139 patients). 16 studies examined oral nabilone, 13 examined oral dronabinol, and 1 examined intramuscular levonantradol. The active control groups were administered prochlorperazine (12 studies), metoclopramide (4 studies), chlorpromazine (2 studies), thiethylperazine (1 study), haloperidol (1 study), domperidone (2 study), and alizapride (1 study). Cannabinoids were more effective than other active treatment drugs for completely relieving nausea and vomiting in the first 24 hours of chemotherapy (table). Cannabinoids also increased both potentially beneficial and harmful additional effects (table).

\section{Conclusion}

In patients having chemotherapy, cannabinoids control nausea and vomiting better than selected conventional antiemetics but are associated with increased side effects.

Cannabis $v$ active antiemetic or placebo at 24 hours in patients with chemotherapy-induced sickness*

\begin{tabular}{|c|c|c|c|c|}
\hline Outcome (number of studies) & Other drug & Weighted event rates & $\mathrm{RBI}(95 \% \mathrm{Cl})$ & NNT (Cl) \\
\hline Complete nausea relief (7) & Active & $59 \% \vee 43 \%$ & $38 \%(18$ to 62$)$ & $7(4$ to 16$)$ \\
\hline Complete vomiting relief (6) & Active & $57 \% \vee 45 \%$ & $28 \%(8$ to 51$)$ & $8(5$ to 38$)$ \\
\hline "High" sensation (8) & Active or placebo & $35 \%$ v 3\% & $10 \%(6$ to 16$)$ & $4(3$ to 4$)$ \\
\hline Drowsiness or sedation (15) & Active or placebo & $50 \% \vee 30 \%$ & $66 \%(46$ to 89$)$ & $5(4$ to 7$)$ \\
\hline \multirow[t]{2}{*}{ Euphoria (3) } & Active & $14 \%$ v $1 \%$ & $12 \%(2$ to 51$)$ & $8(6$ to 12$)$ \\
\hline & & & $\mathrm{RRI}(\mathrm{Cl})$ & NNH (Cl) \\
\hline Dizziness (9) & Active or placebo & $49 \% \vee 17 \%$ & $3 \%(1$ to 3$)$ & $4(3$ to 4$)$ \\
\hline Dysphoria or depression (10) & Active or placebo & $13 \% \vee 0.3 \%$ & $7 \%(2$ to 18$)$ & $9(7$ to 12$)$ \\
\hline Hallucination (10) & Active or placebo & $6 \% \vee 0 \%$ & $\infty$ & 17 (12 to 27$)$ \\
\hline Paranoia (6) & Active or placebo & $5 \% \vee 0 \%$ & $\infty$ & 20 (13 to 42$)$ \\
\hline Hypotension (13) & Active or placebo & $25 \% \vee v 11 \%$ & $1 \%(1$ to 2$)$ & $8(6$ to 11$)$ \\
\hline
\end{tabular}

${ }^{*}$ Abbreviations defined in glossary; RBI and RRI calculated from data in article using a fixed-effects model; NNT, NNH, and $\mathrm{Cl}$ provided in article.

COMMENTARY_continued from previous page

Of greater importance is the observation that patients often expressed a preference for the cannabinoids over other antiemetics for future chemotherapy despite the related psychotropic effects. This observation cannot be ignored. One can only speculate whether patient preference was a result of the beneficial side effects, such as euphoria, or another unknown effect. Irrespective of the reason, patient preference should be included as a valid outcome in the design of future studies that evaluate the efficacy of cannabinoids in the treatment of chemotherapy-induced nausea and vomiting.

$$
\begin{array}{r}
\text { Barry M Kinzbrunner, MD } \\
\text { Vitas Healthcare Corporation, Miami, Florida, USA }
\end{array}
$$

1 Voth EA, Schwartz RH. Medicinal applications of delta-9-tetrahydrocannibinol and marijuana. Ann Intern Med 1997;126:791-8.

2 Nelson KA, Walsh D, Sheehan FA. The cancer-anorexia cachexia syndrome. J Clin Oncol 1994;12:213-25.

3 Meek C. Doctors want cannabis prescriptions allowed. BMA News Review1994;February:1.

4 Belknap S. Commentary on "Review: Delta-9-tetrahydrocannabinol is effective in alleviating cancer chemotherapy induced nausea." ACP J Club 1997 Nov-Dec;127:65. Comment on: Voth EA, Schwartz RH. Medicinal applications of delta-9-tetrahydrocannabinol and marijuana. Ann Intern Med 1997;126:791-8.
Sources of funding: Swiss National Science Foundation and Royal College of Nursing Institute.

For correspondence: $D r$ M R Tramèr, Division d'Anesthésiologie, Département

Anesthésiologie, Pharmacologie Clinique et Soins Intensif de Chirurgie, Hôpitaux Universitaries, CH-1211 Geneva 14, Switzerland. Martin.Tramer@hcuge.ch. 